\title{
Las causas del absentismo de los estudiantes de Derecho según su propia opinión
}

\author{
Law student views on the reasons for their class \\ absenteeism
}

\begin{abstract}
Max Turull
Profesor Titular de Universidad. Departamento de Historia del Derecho, Derecho Romano y Derecho Eclesiástico del Estado, Facultad de Derecho, Universidad de Barcelona. Secretario del Instituto de Desarrollo Profesional (IDP-ICE) de la Universidad de Barcelona (España).

E-mail: mturull@ub.edu
\end{abstract}

\section{Berta Roca}

Licenciada en Psicopedagogía. Técnica de Calidad, Facultad de Derecho, Universidad de Barcelona (España). E-mail: bertaroca@ub.edu

\section{Pilar Aparicio-Chueca}

Profesora Titular de Universidad. Departamento de Empresa, Facultad de Economía y Empresa, Universidad de Barcelona (España).

E-mail: pilaraparicio@ub.edu

\section{Xavier Triadó-Ivern}

Profesor Titular de Universidad. Departamento de Empresa, Facultad de Economía y Empresa, Universidad de Barcelona. Director del Instituto de Desarrollo Profesional (IDP-ICE) de la Universidad de Barcelona (España).

E-mail: xtriado@ub.edu

\section{Joan Guardia-Olmos}

Profesor Catedrático de Universidad. Departamento de Psicología Social y Psicología Cuantitativa, Sección de Psicología Cuantitativa, Facultad de Psicología, Universidad de Barcelona. Instituto de Neurociencias. UB Instiute of Complex Systems. Instituto de Desarrollo Profesional (IDP-ICE) (España). E-mail: jguardia@ub.edu

\section{Pilar Presas}

Departamento de Organitzación, Gestión Empresarial y Diseño de Producto. Escuela Politécnica Superior. Universidad de Gerona.

E-mail: pilar.presas@ub.edu

\section{Mercè Bernardo}

Profesora Agregada Serra Hunter. Departamento de Empresa, Facultad de Economía y Empresa, Universidad de Barcelona (España).

E-mail: merce.bernardo@ub.edu

\section{Amal Elasri-Ejjaberi}

Departamento de Economía y Empresa. Facultad de Empresa y Comunicación. Universidad de VicUniversidad Central de Cataluña (España).

E-mail: amal.elasri@ub.edu

\section{Irene Maestro-Yarza}

Profesora Titular de Universidad. Departamento de Historia Económica, Instituciones, Política y Economía Mundial, Facultad de Economía y Empresa, Universidad de Barcelona (España).

E-mail: imaestro@ub.edu 
Resumen: El absentismo en las aulas de los estudiantes de Derecho se ha convertido recientemente en un lugar común de las preocupaciones del profesorado y de la administración universitaria. El objetivo del presente estudio, que cuenta con precedentes en los cursos 2007-2008 y 2009-2010, es identificar los motivos esgrimidos por los estudiantes del grado de Derecho de la UB para explicar las causas del fenómeno absentista. El trabajo, más allá de su vertiente científica, pretende convertirse en un instrumento para poder diseñar políticas, sobre todo docentes y académicas, para reducir el absentismo en aquellos ámbitos en que sea posible. El estudio se ha realizado a partir de la opinión recogida a una muestra de 577 estudiantes del grado durante la primavera del 2017 , mediante un muestreo aleatorio simple y a través de un cuestionario con 18 preguntas cerradas sobre los motivos que llevan a los estudiantes a no asistir a clase, 5 variables sociodemográficas y una pregunta abierta final. Se ha realizado un análisis estadístico cuantitativo, un análisis factorial y un análisis cualitativo de las respuestas abiertas. Los resultados indican que los posibles factores vinculados con el absentismo tienen que ver, en un sentido amplio, con la metodología docente - en muchos casos todavía únicamente de tipo magistral- utilizada por los profesores; no son irrelevantes las causas debidas a la organización logística del estudiante, pero en todo caso son causas secundarias. Que los profesores dispongan de otros recursos metodológicos además de la clase magistral y saber otorgar valor añadido a las clases desarrollando por tanto políticas docentes y formación del profesorado- se antojan como algunas de las soluciones a un fenómeno complejo y multicausal.

Palabras clave: Absentismo, estudios universitarios, estudiantes Derecho, Asistencia, política docente, EEES.

Abstract: Absenteeism among Law School students is a frequent concern among both university faculty and administrators. The aim of this study, building on previous surveys undertaken in academic years 2007-2008 and 2009-2010 years, is to identify the reasons given by students matriculated on the undergraduate degree in Law at the University of Barcelona to account for class absenteeism. The study, as well as undertaking a rigorous academic analysis, seeks to develop a pedagogical and academic policy tool for reducing absenteeism as and where possible. The study is based on the opinions of 577 undergraduate students collected in the spring of 2017 by simple random sampling. The students completed a questionnaire in which they responded to 
18 closed questions and a final open question addressing the reasons for their nonattendance. The questionnaire also collected information in relation to five sociodemographic variables. A quantitative statistical analysis, factor analysis and qualitative analysis were carried out on the responses to the open question. Broadly speaking, the results indicate that absenteeism is related to the teaching methodology employed by university lecturers - in many instances still overly lecture-based and teacher-centred. Students also mention the conflicting problems of logistical organization that they face, and while not an irrelevant factor, it can in all circumstances be considered a secondary cause. Among the solutions suggested for rectifying this complex, multi-causal phenomenon are the need for teachers to implement other methodological resources in addition to the traditional lecture-based class and to develop an ability to endow their classes with added value - targets that will require the promotion of teacher policies and teacher training.

Keywords: Absenteeism, higher education studies, Law school students, attendance, teaching policy, EHEA

\section{Las causas del absentismo de los estudiantes de Derecho según su propia opinión.}

Education Law student views on the reasons for their class absenteeism.

\section{Introducción}

El absentismo constituye un problema común y en continuo crecimiento en la Universidad de hoy, convirtiéndose en una de las principales preocupaciones de las autoridades académicas. Conlleva un desaprovechamiento de recursos públicos y contradice algunas de las premisas del modelo de enseñanza y aprendizaje del Espacio Europeo de Educación Superior (EEES).

En una universidad presencial el absentismo estudiantil indica no tan solo un divorcio entre enseñanza y aprendizaje, sino cierta patología en el proceso mismo de aprendizaje. Si se está convencido de que la relación directa y presencial entre docente y estudiante infiere un valor en el proceso de aprendizaje, debemos conocer y comprender por qué se produce la divergencia más radical entre ambos actores. Solamente identificando y comprendiendo el absentismo universitario estaremos en disposición de adoptar las medidas convenientes para reducirlo en los ámbitos que nos son propios. 
Durante los cursos 2007-2008 y 2009-2010 la Facultad de Derecho de la Universidad de Barcelona llevó a cabo un estudio sobre el absentismo en los primeros cursos del título de Derecho (GESOP 2008, 2010; Turull y Roca, 2012). En aquella ocasión se entrevistaron telefónicamente a todos los estudiantes de primer curso. Los resultados de este primer estudio, del curso 2007-08, concluían que la mitad de los estudiantes entrevistados asistían a clase siempre o casi siempre. En cambio, una cuarta parte iba a las aulas a veces $(27,9 \%)$, mientras que dos de cada diez asistían a clase poco o nada. Los resultados del estudio del curso 2009-10 no difieren mucho del anterior: el 70,1\% de los estudiantes entrevistados asistían siempre o casi siempre a clase, mientras que un $20,2 \%$ lo hacían a veces y un $5,0 \%$ iba poco o nada. El 4,8\% restante manifestaba haber abandonado los estudios. Aunque no disponemos de estudios más recientes sobre la cuantificación de este fenómeno, la percepción general entre el profesorado es que el absentismo ha aumentado considerablemente a lo largo de los últimos dos o tres años.

Los resultados del estudio del curso 2007-08, todavía para licenciatura, habían identificado como principales motivos, la metodología empleada, el profesor, la dificultad y la falta de motivación. En el curso 2009-10, ya con los nuevos grados implantados, los factores más identificados por los estudiantes seguían siendo la metodología y el profesor, juntamente con el de preparase la materia por su cuenta, causa que no había sido registrada en el anterior curso.

Ahora presentamos los resultados de un tercer estudio realizado durante el curso 20172018. En este caso contemplamos 22 factores que se agrupaban en cinco factores asociados al absentismo: "preparar la materia por su cuenta", "profesor y metodología", “no poder asistir", "falta de motivación” y "otros".

Teniendo en cuenta los dos precedentes mencionados y la literatura sobre el tema, nuestra voluntad es confirmar, o refutar, los resultados obtenidos $\mathrm{y}$, sobretodo, identificar con los grados del EEES ya asentados y con un nuevo perfil de estudiantes, los principales factores asociados al fenómeno del absentismo en los estudios de Derecho a partir de la opinión de los propios actores a fin de intentar combatir los motivos que realmente sean "combatibles". 


\section{Revisión bibliográfica}

Aunque existen pocos estudios empíricos sobre el fenómeno del absentismo, la revisión de la literatura nos muestra una creciente preocupación y en consecuencia un aumento del número de investigaciones. Según López Bonilla y López Bonilla (2015), se distinguen tres enfoques en los estudios e investigaciones sobre el absentismo. Aquellos estudios que tienen como principal objetivo establecer las causas del absentismo (Devadoss y Foltz, 1996; Garrido y Sacristán, 2010; Gracia e Iglesia, 2007; Longhurst, 1999; Paisey y Paisey, 2004; Reid, 2008; Rodríguez, 2010; Rodríguez et al., 2003; Romer, 1993; Triadó-Ivern et al., 2009), otros que focalizan su interés en las consecuencias del absentismo, y por lo tanto en la relación entre absentismo y rendimiento académico (Durden y Ellis, 1995; Marburger, 2001, 2006; McCarey, Barr, y Rattray, 2007; Romer, 1993), y algunos que se centran en la solución del problema a través de la definición de programas preventivos y/o correctivos (Marburger, 2006; Ruiz, Ceballos, y García, 2010; Triadó-Ivern, 2009). Aunque el enfoque de los estudios es mayoritariamente cuantitativo, encontramos algunas investigaciones con una aproximación más cualitativa (Moore et al, 2008 y Clores, 2009).

Tabla 1: Literatura científica invocada en el artículo

\begin{tabular}{|l|l|}
\hline Enfoque & Autores \\
\hline Establecer las causas del absentismo & Devadoss y Foltz, 1996 \\
& Garrido y Sacristán, 2010 \\
& Gracia e Iglesia, 2007 \\
& Longhurst, 1999 \\
& Paisey y Paisey, 2004 \\
& Reid, 2008 \\
& Rodríguez, 2010 \\
& Rodríguez et al., 2003 \\
& Romer, 1993 \\
& Triadó-Ivern et al. 2009 \\
\hline $\begin{array}{l}\text { Consecuencias del absentismo (por } \\
\text { ejemplo relación entre absentismo y } \\
\text { rendimiento académico) }\end{array}$ & Durden y Ellis, 1995 \\
& Marburger, 2001, 2006 \\
& McCarey, Barr, y Rattray, 2007 \\
\hline $\begin{array}{l}\text { Buscar soluciones al problema a } \\
\text { través de la definición de programas } \\
\text { preventivos y/o correctivos }\end{array}$ & Marburger, 2006 \\
& Ruiz, Ceballos, y García, 2010 \\
& Triadó-Ivern 2009 \\
\hline
\end{tabular}

Estudios pioneros como el de Romer (1993) ya se cuestionaban el alcance del absentismo en las aulas y como éste afectaba al rendimiento académico. Su investigación concluyó que un tercio de les estudiantes no asistían a clase, y sugirió que la asistencia puede afectar sustancialmente al aprendizaje. En esta misma línea se 
encuentra el estudio realizado por Cabrera, Bethencourt, González y Álvarez (2016) que corroboró que la asistencia regular a clase impactaba en el hecho de terminar los estudios en el tiempo establecido. Marburguer (2001) estudió la relación de la asistencia a clase con el rendimiento de las pruebas de los contenidos de estas clases. Los resultados exponen que el rendimiento de las pruebas aumenta con la asistencia, aunque esta mejora es relativamente pequeña.

Respecto a la identificación de las causas, varios autores han intentado identificar las razones que llevan a los estudiantes a no asistir a clase a través de las opiniones que ellos mismos manifiestan. Por ejemplo, Triadó-Ivern et al. (2013) distingue 12 causas del absentismo de los estudiantes de la Facultad de Economía y Empresa de la Universidad de Barcelona, que finalmente se agrupan en cuatro factores relacionados con: las características de los profesores y de las asignaturas, los elementos estructurales (horarios, superposición de clases.), los recursos materiales disponibles para los estudiantes (manual, apuntes), y las características del curso en que la asistencia no era obligatoria.

Por otra parte, López-Bonilla y López-Bonilla (2015) determinan la metodología docente como el motivo principal del absentismo de los estudiantes de Turismo de la Universidad de Sevilla. El estudio contempla el análisis de 28 factores determinantes que se agruparon en siete principales causas que atienden a la eficacia, el estilo docente, el interés académico, los contenidos y formatos docentes, la influencia y temores de clase, imponderables y de conveniencia.

También Rodríguez et al. (2003) realizó un estudio para conocer los motivos del absentismo, cuyos resultados indicaron como factores la organización académica, la metodología, la utilidad de las lecciones o la actitud de los profesores. Más adelante, Rodríguez establecería una clasificación de las causas del absentismo en dos tipos de categorías: académicas y extraacadémicas. "Se entiende como académicas aquellas que se producen en el seno de la misma universidad, que pertenecen a su contexto, por normas o por regulaciones. Contrariamente, las causas extraacadémicas pertenecen al exterior de la universidad y obedecen a muy diferentes orígenes ajenos" (Rodríguez, 2010).

Garrido y Sacristán (2010) agrupan los factores ligados al absentismo en cuatro categorías: profesor, alumno, materia y organización. También destacan como factores 
especialmente relevantes: 1) el elevado número de convocatorias por asignatura; 2) el orden de elección de centro al hacer la matricula; y 3) otras características del sistema.

Ante la certeza, señalada por la literatura y por nuestro propio estudio, de la complejidad del fenómeno, una buena comprensión del mismo se antoja necesaria para abordar las soluciones más eficaces desde los más variados ámbitos de actuación.

\section{Método}

\subsection{Participantes}

El estudio se llevó a cabo a partir de la opinión recogida a una muestra de 577 estudiantes del grado en Derecho de la Universidad de Barcelona durante la primavera del 2017 mediante un muestreo aleatorio simple. Se obtuvieron 577 cuestionarios con 119 respuestas abiertas.

\subsection{Instrumento}

El fenómeno del absentismo universitario es un problema real, multidimensional, complejo y nada fácil de medir. La dificultad radica en cómo conseguir respuestas de "estudiantes que no asisten a clase y estudiantes ausentes". Teniendo en cuenta esta limitación, se ha optado por realizar un cuestionario respaldado por la literatura (Triadó, Aparicio, Guàrdia, Peró i Jaría, 2013) y preguntar a los estudiantes que asisten a clase por los motivos por los cuales sus compañeros de clase no asisten. De esta manera, se intentó recoger la información sobre cada sujeto de manera veraz y eliminar el miedo a responder en primera persona.

El cuestionario plantea 18 preguntas cerradas sobre los motivos que llevan a los estudiantes a no asistir a clase, 5 variables sociodemográficas y una pregunta abierta final. Se ha utilizado una escala Likert que varía desde 1 (totalmente en desacuerdo) hasta 4 (totalmente de acuerdo). El análisis de fiabilidad toma un valor correcto, obteniendo una alfa de Cronbach de 0,70, y fue estadísticamente significativa para la prueba T-cuadrado de Hotelling y la prueba de aditividad de Tukey.

\subsection{Procedimiento}

En una primera fase el equipo de investigación seleccionó las asignaturas y los grupos en los que se realizaría la encuesta, teniendo en cuenta que debían abarcar todos los cursos (de primero a cuarto) y los dos turnos horarios (mañana y tarde). En segundo lugar, los dos miembros del equipo adscritos a la Facultad de Derecho se ocuparon de contactar personalmente con los profesores que impartían los grupos y asignaturas 
seleccionadas para solicitarles que pasaran el cuestionario entre sus alumnos durante su hora de clase; el cuestionario se pasó a un total de 13 grupos. Se programaron dos semanas para distribuir y recuperar todos los cuestionarios cumplimentados. Fueron trasladados a dos miembros del equipo que procesaron todos los datos. La tabla 2 muestra el perfil sociodemográfico de los encuestados.

Tabla 2: Perfil sociodemográfico de los estudiantes

\begin{tabular}{|c|c|c|}
\hline & & Porcentaje \\
\hline \multirow[t]{2}{*}{ Sexo } & Hombre & 35,5 \\
\hline & Mujer & 63,0 \\
\hline \multirow[t]{4}{*}{ Curso } & $1^{\circ}$ & 31,8 \\
\hline & $2^{\circ}$ & 30,2 \\
\hline & $3^{\circ}$ & 24,1 \\
\hline & $4^{\circ}$ & 13,4 \\
\hline \multirow[t]{2}{*}{ Turno } & Mañana & 90,1 \\
\hline & Tarde & 8,6 \\
\hline
\end{tabular}

\subsection{Análisis de datos}

Para el análisis cuantitativo, teniendo en cuenta la procedencia de los datos y la escala del fenómeno, se realizó un análisis de comparación de medias y posteriormente se llevó a cabo un análisis factorial exploratorio para identificar la dimensionalidad del mismo (el análisis factorial exploratorio siguió el método de componentes principales con rotación oblimin y se extrajeron aquellos factores con autovalores mayores que la unidad). Los valores de alfa de Cronbanch oscilaron entre el 0,697 y un índice de KMO de 0,714 , lo que indica una consistencia interna correcta de los ítems que miden cada constructo y estadísticamente significativa para la prueba T-cuadrado de Hotelling y la prueba de aditividad de Tukey.

En cuanto a la muestra de preguntas abiertas, fueron analizadas y codificadas mediante el paquete de software ATLAS.ti.

\section{Resultados}

\subsection{Análisis cuantitativo}

La tabla 3 muestra las razones del absentismo a partir de las opciones ofrecidas en la encuesta. El motivo $1^{\circ}$ que con diferencia ofrece mayor adhesión es "la forma de explicar del profesor", con una media de 3,25 sobre 4, una de las menores desviaciones 
estándar de 0,722, y uno de los mayores números de respuestas en términos absolutos (600). En $2^{\circ}$ lugar la opinión de que "las clases son pesadas", con 2,89, una desviación igualmente baja de 0,739 y el mayor número de respuestas absolutas (602). En tercer $\left(3^{\circ}\right)$ lugar la opinión de que el motivo es que "no se obliga a ir a clase" con 2,78 y variación de 0,980. En $4^{\circ}$ que "van a evaluación única (o final)" con 2,77, aunque con desviación de 1,032. En $5^{\circ}$ lugar que "el profesor dicta apuntes o lee transparencias" con

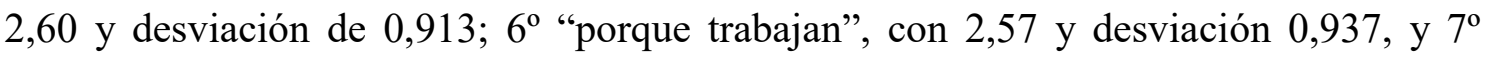
porque "ya disponen de buenos apuntes", con mediana de 2,53 y desviación de 0,917.

En el sentido opuesto, también conviene señalar aquellos factores, con medias inferiores a 2, que según los encuestados no favorecen ni explican el absentismo. Pocos creen que el absentismo se deba a que "las academias (centros de estudios privados externos a la facultad) son mejores para aprobar", con tan solo mediana de 1,40 y la más baja desviación de 0,686 , lo que muestra un consenso considerable, o a que "el contenido sea fácil", con mediana de 1,87 y variación débil de 0,748, o a que han "matriculado demasiados créditos", con mediana 1,99.

La misma Tabla 3 ofrece resultados más pormenorizados según el género, el curso y el turno de clase en que estaban matriculados los encuestados. Atendiendo al género las diferencias son poco significativas. Hay coincidencia en que los principales motivos son "la forma de explicar" (3,17 en hombres y 3,30 en mujeres) y que "las clases son pesadas" (2,83 y 2,92). Se percibe una leve diferencia a partir de aquí: mientras en tercer lugar los hombres se decantan a que "van a evaluación única (o final)" $(2,80)$, las mujeres creen que es debido a que "no se obliga a ir a clase" $(2,81)$; estas opiniones se invierten en cuarto lugar. La única diferencia estadísticamente significativa radica en que "el contenido no interesa", significativamente mayor en hombres que en mujeres.

En cambio, más sugerente se presenta la opinión según el curso de los encuestados. Atendiendo únicamente a las diferencias estadísticamente significativas (marcadas con asterisco en F en la tabla 3), se observan dos lógicas distintas. En primer lugar, cuando la diferencia de opinión no se halla tanto entre el bloque $1^{\circ}$ y $2^{\circ}$ curso versus $3^{\circ}$ y $4^{\circ}$, sino más bien de $1^{\circ}$ a $2^{\circ}$ : los alumnos noveles, de primer curso, estiman significativamente más que los de segundo curso que "ya hay suficiente material" y que "el profesor dicta apuntes o lee transparencias". En cambio resulta a la inversa cuando son los estudiantes más veteranos los que invocan, para no ir a clase, que en su caso ya disponen de buenos apuntes. Efectivamente una cosa es disponer de "material" 
(manuales, transparencias, etc.) como invocan los de $1^{\circ}$, y otra distinta es disponer de “apuntes". Y en segundo lugar, las principales diferencias entre $1^{\circ}$ y $2^{\circ}$ respecto $3^{\circ}$ y $4^{\circ}$ se hallan, como es natural, cuando se alude a "repetir asignatura", "solapamiento de horarios", "simultaneidad de estudios", "demasiados créditos matriculados" o "motivos laborales". Pero también, significativamente, en que "las clases son pesadas": para los alumnos de cursos superiores, con mayor madurez intelectual y académica, este motivo pierde peso.

Las variaciones en la percepción de las causas del absentismo si atendemos al turno de los encuestados, según sea de mañana o tarde (tabla 3), son mínimas. Se aprecia un elevado consenso en muchas de las respuestas: "contenido no interesa", "ir a clase no sirve para aprobar", es "más útil estudiar en la biblioteca" o "ya disponen de buenos apuntes". La única diferencia significativa atiende a razones de "logística": los estudiantes del turno de tarde ponen más énfasis que los de mañana en que "trabajan", "simultanean estudios" o han matriculado "demasiados créditos". 
Tabla 3: Percepción de los estudiantes de por qué sus compañeros no asisten a clase.

\begin{tabular}{|c|c|c|c|c|c|c|c|c|c|c|c|c|}
\hline & General & Gér & & & $\mathbf{1}^{\text {st }}$ & $2^{\text {nd }}$ & $\mathbf{3}^{\text {rd }}$ & $4^{\text {th }}$ & & Mañana & Tarde & \\
\hline & $\begin{array}{c}\text { Media } \\
(D E)\end{array}$ & $\begin{array}{c}\text { Hombre } \\
(D E)\end{array}$ & $\begin{array}{l}\text { Mujer } \\
(D E)\end{array}$ & $t$ & $\begin{array}{l}\text { Media } \\
(D E)\end{array}$ & $\begin{array}{c}\text { Media } \\
(D E)\end{array}$ & $\begin{array}{c}\text { Media } \\
(D E)\end{array}$ & $\begin{array}{l}\text { Media } \\
(D E)\end{array}$ & $F$ & $\begin{array}{c}\text { Media } \\
(D E)\end{array}$ & $\begin{array}{l}\text { Media } \\
(D E)\end{array}$ & $F$ \\
\hline Clases pesadas & $\begin{array}{l}2,89 \\
(, 739) \\
\end{array}$ & $\begin{array}{l}2,83 \\
(, 791) \\
\end{array}$ & $\begin{array}{l}2,92 \\
(, 706)\end{array}$ & $-1,54$ & $\begin{array}{l}2,92 \\
(, 697)\end{array}$ & $\begin{array}{l}3,08 \\
(, 698)\end{array}$ & $\begin{array}{l}2,79 \\
(, 733)\end{array}$ & $\begin{array}{l}2,59 \\
(, 807)\end{array}$ & $\begin{array}{c}9,93 \\
*\end{array}$ & $\begin{array}{c}2,91 \\
(, 733) \\
\end{array}$ & $\begin{array}{c}2,78 \\
(, 764) \\
\end{array}$ & 1,46 \\
\hline Contenido no interesa & $\begin{array}{l}2,24 \\
(, 705)\end{array}$ & $\begin{array}{l}2,34 \\
(, 722)\end{array}$ & $\begin{array}{l}2,18 \\
(, 693)\end{array}$ & $\begin{array}{c}2,67 \\
*\end{array}$ & $\begin{array}{l}2,22 \\
(, 712)\end{array}$ & $\begin{array}{l}2,32 \\
(, 667)\end{array}$ & $\begin{array}{l}2,14 \\
(, 703)\end{array}$ & $\begin{array}{l}2,35 \\
(, 734)\end{array}$ & 2,52 & $\begin{array}{l}2,25 \\
(, 699)\end{array}$ & $\begin{array}{l}2,25 \\
(, 771)\end{array}$ &, 004 \\
\hline Contenido sencillo & $\begin{array}{l}1,87 \\
(, 748)\end{array}$ & $\begin{array}{l}1,94 \\
(, 722)\end{array}$ & $\begin{array}{l}1,83 \\
(, 733)\end{array}$ & 1,71 & $\begin{array}{l}1,80 \\
(, 749)\end{array}$ & $\begin{array}{l}1,85 \\
(, 721)\end{array}$ & $\begin{array}{l}1,94 \\
(, 766)\end{array}$ & $\begin{array}{l}1,95 \\
(1,95)\end{array}$ & 1,32 & $\begin{array}{l}1,86 \\
(, 744)\end{array}$ & $\begin{array}{l}2,02 \\
(, 777)\end{array}$ & 2,04 \\
\hline Repiten asignatura & $\begin{array}{l}2,16 \\
(, 936)\end{array}$ & $\begin{array}{l}2,13 \\
(, 899)\end{array}$ & $\begin{array}{l}2,17 \\
(, 955)\end{array}$ &,- 44 & $\begin{array}{l}2,14 \\
(, 946)\end{array}$ & $\begin{array}{l}2,13 \\
(, 946)\end{array}$ & $\begin{array}{l}2,20 \\
(, 940)\end{array}$ & $\begin{array}{l}2,18 \\
(, 934)\end{array}$ &, 17 & $\begin{array}{l}2,14 \\
(, 926)\end{array}$ & $\begin{array}{c}2,35 \\
(1,011)\end{array}$ & 2,17 \\
\hline Ir a clase no sirve para aprobar & $\begin{array}{l}2,03 \\
(, 929)\end{array}$ & $\begin{array}{l}2,07 \\
(, 897)\end{array}$ & $\begin{array}{l}2,01 \\
(, 944)\end{array}$ & ,731 & $\begin{array}{l}2,03 \\
(, 908)\end{array}$ & $\begin{array}{l}2,01 \\
(, 932)\end{array}$ & $\begin{array}{l}2,06 \\
(, 915)\end{array}$ & $\begin{array}{c}2,06 \\
(1,017)\end{array}$ &, 11 & $\begin{array}{l}2,04 \\
(, 929)\end{array}$ & $\begin{array}{c}2,02 \\
(1,000)\end{array}$ &, 017 \\
\hline La manera de explicar & $\begin{array}{l}3,25 \\
(, 722) \\
\end{array}$ & $\begin{array}{l}3,17 \\
(, 772) \\
\end{array}$ & $\begin{array}{l}3,30 \\
(, 688) \\
\end{array}$ & $-2,03$ & $\begin{array}{l}3,19 \\
(, 730) \\
\end{array}$ & $\begin{array}{l}3,38 \\
(, 669) \\
\end{array}$ & $\begin{array}{r}3,23 \\
(, 705) \\
\end{array}$ & $\begin{array}{l}3,15 \\
(, 786) \\
\end{array}$ & 2,82 & $\begin{array}{l}3,27 \\
(, 707) \\
\end{array}$ & $\begin{array}{l}3,16 \\
(, 817) \\
\end{array}$ & 1,05 \\
\hline Ya hay suficiente material & $\begin{array}{l}2,25 \\
(, 913) \\
\end{array}$ & $\begin{array}{l}2,32 \\
(, 919) \\
\end{array}$ & $\begin{array}{l}2,21 \\
(, 911)\end{array}$ & 1,46 & $\begin{array}{l}2,42 \\
(, 881) \\
\end{array}$ & $\begin{array}{l}2,02 \\
(, 868) \\
\end{array}$ & $\begin{array}{l}2,26 \\
(, 926) \\
\end{array}$ & $\begin{array}{l}2,32 \\
(, 981) \\
\end{array}$ & $\begin{array}{c}6,57 \\
* \\
\end{array}$ & $\begin{array}{l}2,26 \\
(, 916) \\
\end{array}$ & $\begin{array}{l}2,04 \\
(, 871) \\
\end{array}$ & 2,83 \\
\hline El profesor dicta apuntes o lee transparencias & $\begin{array}{l}2,60 \\
(, 913)\end{array}$ & $\begin{array}{l}2,66 \\
(, 912)\end{array}$ & $\begin{array}{l}2,57 \\
(, 908)\end{array}$ & 1,12 & $\begin{array}{l}2,43 \\
(, 907)\end{array}$ & $\begin{array}{l}2,73 \\
(, 906)\end{array}$ & $\begin{array}{l}2,63 \\
(, 888)\end{array}$ & $\begin{array}{l}2,64 \\
(, 939)\end{array}$ & $\begin{array}{c}3,63 \\
*\end{array}$ & $\begin{array}{l}2,57 \\
(, 903)\end{array}$ & $\begin{array}{c}2,80 \\
(1,020)\end{array}$ & 2,97 \\
\hline No se obliga a ir a clase & $\begin{array}{l}2,78 \\
(, 980) \\
\end{array}$ & $\begin{array}{c}2,75 \\
(1,018) \\
\end{array}$ & $\begin{array}{l}2,81 \\
(, 954)\end{array}$ &,- 63 & $\begin{array}{l}2,79 \\
(, 987) \\
\end{array}$ & $\begin{array}{c}2,85 \\
(1,025) \\
\end{array}$ & $\begin{array}{l}2,74 \\
(, 901)\end{array}$ & $\begin{array}{c}2,70 \\
(1,017)\end{array}$ &, 53 & $\begin{array}{l}2,80 \\
(, 982)\end{array}$ & $\begin{array}{l}2,65 \\
(, 948) \\
\end{array}$ & ,980 \\
\hline Más útil estudiar en la biblioteca & $\begin{array}{l}2,30 \\
(, 869) \\
\end{array}$ & $\begin{array}{l}2,31 \\
(, 900) \\
\end{array}$ & $\begin{array}{l}2,29 \\
(, 853) \\
\end{array}$ & 20 & $\begin{array}{l}2,26 \\
(, 856) \\
\end{array}$ & $\begin{array}{l}2,38 \\
(, 911) \\
\end{array}$ & $\begin{array}{l}2,24 \\
(, 792) \\
\end{array}$ & $\begin{array}{l}2,31 \\
(, 936) \\
\end{array}$ & ,92 & $\begin{array}{l}2,30 \\
(, 868) \\
\end{array}$ & $\begin{array}{l}2,28 \\
(, 904) \\
\end{array}$ &, 025 \\
\hline Academias mejor para aprobar & $\begin{array}{l}1,40 \\
(, 686) \\
\end{array}$ & $\begin{array}{l}1,35 \\
(, 650) \\
\end{array}$ & $\begin{array}{l}1,44 \\
(, 709) \\
\end{array}$ & $-1,34$ & $\begin{array}{l}1,35 \\
(, 623)\end{array}$ & $\begin{array}{l}1,43 \\
(, 685) \\
\end{array}$ & $\begin{array}{c}1,31 \\
(, 555) \\
\end{array}$ & $\begin{array}{l}1,62 \\
(, 946) \\
\end{array}$ & $\begin{array}{c}4,01 \\
* \\
\end{array}$ & $\begin{array}{c}1,39 \\
(, 671) \\
\end{array}$ & $\begin{array}{c}1,53 \\
(, 819) \\
\end{array}$ & 1,86 \\
\hline Ya disponen de buenos apuntes & $\begin{array}{c}2,53 \\
(, 917) \\
\end{array}$ & $\begin{array}{c}2,45 \\
(, 906)\end{array}$ & $\begin{array}{c}2,57 \\
(, 925)\end{array}$ & $-1,45$ & $\begin{array}{c}2,23 \\
(, 858)\end{array}$ & $\begin{array}{l}2,65 \\
(, 934) \\
\end{array}$ & $\begin{array}{l}2,66 \\
(, 912) \\
\end{array}$ & $\begin{array}{c}2,72 \\
(, 876)\end{array}$ & $\begin{array}{c}10,35 \\
*\end{array}$ & $\begin{array}{c}2,53 \\
(, 920) \\
\end{array}$ & $\begin{array}{c}2,55 \\
(, 843) \\
\end{array}$ & ,026 \\
\hline Van a evaluación única & $\begin{array}{c}2,77 \\
(1,032)\end{array}$ & $\begin{array}{c}2,80 \\
(1,068)\end{array}$ & $\begin{array}{c}2,76 \\
(1,008)\end{array}$ & 46 & $\begin{array}{c}2,72 \\
(1,092)\end{array}$ & $\begin{array}{c}2,86 \\
(1,007) \\
\end{array}$ & $\begin{array}{l}2,84 \\
(, 983) \\
\end{array}$ & $\begin{array}{c}2,53 \\
(1,021)\end{array}$ & 2,21 & $\begin{array}{c}2,77 \\
(1,030)\end{array}$ & $\begin{array}{c}2,85 \\
(1,000)\end{array}$ & ,267 \\
\hline Solapamiento de horarios & $\begin{array}{c}2,23 \\
(1,034)\end{array}$ & $\begin{array}{c}2,13 \\
(1,073)\end{array}$ & $\begin{array}{c}2,30 \\
(1,010)\end{array}$ & $-1,78$ & $\begin{array}{c}1,89 \\
(, 994) \\
\end{array}$ & $\begin{array}{c}2,05 \\
(, 965) \\
\end{array}$ & $\begin{array}{c}2,55 \\
(1,001) \\
\end{array}$ & $\begin{array}{c}2,79 \\
(, 951) \\
\end{array}$ & $\begin{array}{c}22,57 \\
* \\
\end{array}$ & $\begin{array}{c}2,20 \\
(1,034)\end{array}$ & $\begin{array}{c}2,49 \\
(, 987) \\
\end{array}$ & 3,75 \\
\hline Simultanean estudios & $\begin{array}{c}2,18 \\
(, 923) \\
\end{array}$ & $\begin{array}{c}2,12 \\
(, 902) \\
\end{array}$ & $\begin{array}{c}2,22 \\
(, 927) \\
\end{array}$ &,- 75 & $\begin{array}{l}1,99 \\
(, 902) \\
\end{array}$ & $\begin{array}{l}2,13 \\
(, 930) \\
\end{array}$ & $\begin{array}{c}2,26 \\
(, 892) \\
\end{array}$ & $\begin{array}{r}2,57 \\
(, 899) \\
\end{array}$ & $\begin{array}{c}7,63 \\
* \\
\end{array}$ & $\begin{array}{r}2,14 \\
(, 927) \\
\end{array}$ & $\begin{array}{r}2,52 \\
(, 825) \\
\end{array}$ & $\begin{array}{c}7,36 \\
* \\
\end{array}$ \\
\hline Demasiados créditos & $\begin{array}{l}1,99 \\
(, 909) \\
\end{array}$ & $\begin{array}{l}1,90 \\
(, 885) \\
\end{array}$ & $\begin{array}{l}2,05 \\
(, 924) \\
\end{array}$ & $-1,02$ & $\begin{array}{c}1,82 \\
(, 840) \\
\end{array}$ & $\begin{array}{r}1,85 \\
(, 891) \\
\end{array}$ & $\begin{array}{r}2,14 \\
(, 930) \\
\end{array}$ & $\begin{array}{l}2,42 \\
(, 905) \\
\end{array}$ & $\begin{array}{c}11,17 \\
*\end{array}$ & $\begin{array}{l}1,95 \\
(, 901) \\
\end{array}$ & $\begin{array}{l}2,31 \\
(, 918) \\
\end{array}$ & $\begin{array}{c}6,78 \\
*\end{array}$ \\
\hline Porque trabajan & $\begin{array}{l}2,57 \\
(, 937) \\
\end{array}$ & $\begin{array}{l}2,47 \\
(, 969) \\
\end{array}$ & $\begin{array}{l}2,63 \\
(, 915) \\
\end{array}$ &,- 98 & $\begin{array}{l}2,38 \\
(, 982) \\
\end{array}$ & $\begin{array}{l}2,55 \\
(, 880) \\
\end{array}$ & $\begin{array}{c}2,62 \\
(, 922) \\
\end{array}$ & $\begin{array}{l}2,90 \\
(, 894) \\
\end{array}$ & $\begin{array}{c}5,94 \\
* \\
\end{array}$ & $\begin{array}{l}2,53 \\
(, 927) \\
\end{array}$ & $\begin{array}{c}3,06 \\
(, 890) \\
\end{array}$ & $\begin{array}{c}15,01 \\
* \\
\end{array}$ \\
\hline Viven lejos & $\begin{array}{l}2,46 \\
(, 958) \\
\end{array}$ & $\begin{array}{l}2,56 \\
(, 969) \\
\end{array}$ & $\begin{array}{r}2,40 \\
(, 915) \\
\end{array}$ & 1,12 & $\begin{array}{l}2,35 \\
(, 942) \\
\end{array}$ & $\begin{array}{l}2,54 \\
(, 998) \\
\end{array}$ & $\begin{array}{r}2,50 \\
(, 899) \\
\end{array}$ & $\begin{array}{l}2,47 \\
(, 996) \\
\end{array}$ & 1,32 & $\begin{array}{c}2,45 \\
(, 961) \\
\end{array}$ & $\begin{array}{l}2,59 \\
(, 956) \\
\end{array}$ & 1,028 \\
\hline
\end{tabular}

REVISTA DE EDUCACIÓN Y DERECHO.EDUCATION AND LAW REVIEW Fecha de entrada: 04-03-2019
Número 19 Octubre 2018-Marzo 2019

Fecha de aceptación: 13-03-2019 
Comparando los motivos de absentismo por edad, no existen diferencias significativas entre los intervalos, pero se ha considerado interesante realizar una comparación entre los estudiantes más jóvenes con los más mayores (gráfico 1). La mayoría de los motivos tienen puntuaciones más elevadas en los estudiantes más jóvenes excepto en las variables de "solapamiento de horarios", "simultaneidad de estudios", "matricularse de demasiados créditos" y "porque trabajan”. Todas estas variables están relacionadas con la edad y por tanto es lógico que exista esta diferencia de percepciones en los motivos de absentismo. En cambio, de manera inversa, las variables sobre metodología docente, como que las clases resultan pesadas, la manera de explicar del profesor, que el contenido no interesa o que ya existe suficiente material fuera del aula, muestran mayor impacto entre los estudiantes jóvenes. Ciertamente, la sensibilidad hacia lo que les empuja al absentismo es diferente.

Gráfico 1: Comparación de los motivos de absentismo entre los dos extremos de edad (18 años y más de 24)

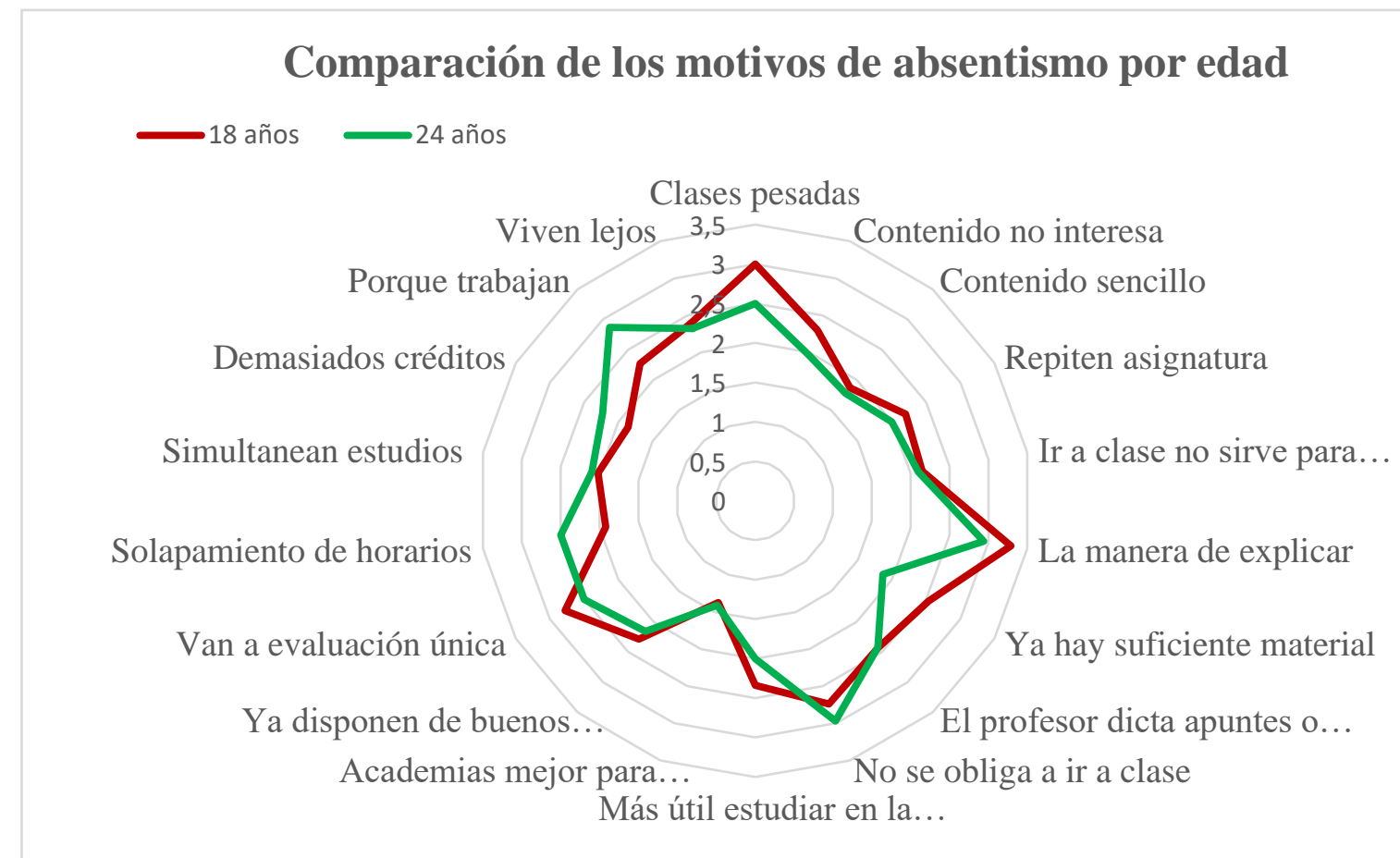

Finalmente (tabla 4) se ha realizado un análisis factorial exploratorio obteniéndose cinco factores, resultando una capacidad explicativa del 50,828\% de la varianza. El primer factor (F1) recoge las variables más directamente vinculadas a la organización y planificación del propio estudiante, como la opción por la evaluación única, solapamiento de horarios, simultaneidad de estudios, matrícula en demasiados créditos, problemas laborales y razones de distancia a la Facultad; este factor muestra una 
capacidad explicativa del $16,98 \%$. El segundo factor describe la utilidad que los estudiantes otorgan a la clase, agrupa las variables vinculadas a la utilidad docente: clases pesadas, el contenido de las asignaturas no interesa, la forma de exponer del profesor o que este dicta apuntes o lee transparencias (explica un 11,78\%,). El tercer factor explica un $8,72 \%$ de la varianza y recoge los motivos de aquellos estudiantes que "van por libre", no están obligados a asistir clase, disponer de los apuntes necesarios y van a evaluación única. El factor cuatro alude a todo lo relacionado con la asignatura, vincula el contenido sencillo, la existencia de material suficiente para prepararla por ellos mismos y que el profesor dicta apuntes o lee transparencias; este factor explica un $7,2 \%$ de la variación del modelo. Y el quinto factor, con una capacidad explicativa del $5,6 \%$, lo forman aquellas variables que incluye todo lo relacionado con el profesorado, por ejemplo "clases pesadas, contenido no interesa, la manera de explicar".

Tabla 4. Matriz de las variables que forma cada factor y su importancia en cada uno de ellos

\begin{tabular}{|l|c|c|c|c|c|}
\hline \multicolumn{5}{|c|}{ Matriz de estructura } \\
\hline & Factor 1 & Factor 2 & Factor 3 & Factor 4 & Factor 5 \\
\hline Clases pesadas & & & & &, 851 \\
\hline Contenido no interesa & & & & &, 784 \\
\hline Contenido sencillo & & & &,- 529 & \\
\hline Repiten asignatura & & & &,- 434 & \\
\hline Ir a clase no sirve para aprobar & &, 738 & & & \\
\hline La manera de explicar & &, 466 & & &, 446 \\
\hline Ya hay suficiente material & & & &,- 718 & \\
\hline $\begin{array}{l}\text { El profesor dicta apuntes o lee } \\
\text { transparencias }\end{array}$ & & & &,- 641 & \\
\hline No se obliga a ir a clase & & &, 663 & & \\
\hline Más útil estudiar en la biblioteca & &, 799 & & & \\
\hline Academias mejor para aprobar & & & & & \\
\hline Ya disponen de buenos apuntes & & &, 735 & & \\
\hline Van a evaluación única & & &, 548 & & \\
\hline Solapamiento de horarios &, 754 & & & & \\
\hline Simultanean estudios &, 830 & & & & \\
\hline Demasiados créditos &, 741 & & & & \\
\hline Porque trabajan &, 779 & & & & \\
\hline Viven lejos &, 469 & & & & \\
\hline
\end{tabular}

En definitiva, pues, "la forma de explicar" del profesor, que "las clases son pesadas", que "no se obliga a ir a clase", que existe la posibilidad de presentarse a "evaluación única", que "el profesor dicta apuntes" y que "ya disponen de buenos apuntes" son los motivos recurrentes según los estudiantes encuestados. 


\subsection{Análisis cualitativo}

Respecto al análisis cualitativo, en primer lugar las preguntas abiertas fueron analizadas y codificadas, identificando diferentes temáticas similares. Las respuestas abiertas que han redactado los estudiantes bajo el epígrafe "otros motivos" en el cuestionario ofrecen un gran abanico de matices sobre los motivos del absentismo en las aulas. Cada estudiante que respondió la encuesta daba su particular punto de vista y su matiz estrictamente personal.

Se han clasificado las más de 100 respuestas abiertas en grupos (tabla 5). Finalmente se establecieron 60 códigos que a su vez se agruparon en 8 familias (metodología docente, profesorado, evaluación, planificación del estudiante, organización docente de la facultad, infraestructura o logística, motivación del estudiante y rasgos del estudiante).

Los grupos más numerosos hacían referencia a las características del profesorado (27 opiniones), a la metodología docente utilizada (19) y a la motivación de los estudiantes (15). En el primer caso, sobre las características del profesorado, un buen número de encuestados aducían a la "[mala] calidad del profesorado" y a que "el profesor no explica bien", además de que "el profesor no motiva", "no incentiva", "no planifica", "no prepara las clases", "no se impone", "no se implica", "no está motivado" o "no interactúa adecuadamente".

Los comentarios abiertos vinculados a la metodología docente aparecen aún más difusos pero apuntan en la misma dirección: "las clases son aburridas", "demasiado teóricas", "poco dinámicas", “innecesarias para aprobar", "poco útiles", "el profesor lee los apuntes" o, directamente, no asisten a causa "de la metodología docente". El conjunto de motivos aducidos que hace referencia a la motivación de los mismos estudiantes reconoce, en primer lugar, "la pereza", "el cansancio", "el sueño" o "el clima" como motivos para ni ir a clase, pero también "la propia desmotivación [del alumno]" (estudian por presión de la familia, o van a cambiar de carrera), "por desinterés", "porque tampoco van los compañeros", "porque no se adaptan" o "porqué supone demasiado esfuerzo".

En un sentido parecido a la motivación estudiantil, pero diferenciable, podemos reconocer las opiniones que hacen referencia a la planificación del propio estudiante (15), algunas de las cuales vinculamos también al sistema de evaluación: "la incompatibilidad entre estudios y actividad laboral", "la matriculación en un doble grado", "porque prefieren dedicar el tiempo a estudiar" tanto si realizan evaluación 
continua como única, "solapamiento de asignatura y coincidencia de exámenes" o "porque les resulta más eficiente preparar la asignatura de manera autónoma que asistiendo a clase".

Tampoco faltan las razones vinculadas a la organización de los estudios (9): "el horario" (con clases que empiezan o demasiado pronto o acaban demasiado tarde), "la duración de las clases" (dos horas se hace pesado, o por una hora ya no van a la Facultad), "grupos masificados", "solapamiento de horarios", "solapamiento de contenidos entre asignaturas", "exceso de trabajo [académico]" o "falta de coordinación entre asignaturas y entre pruebas de evaluación continua". Ciertamente, los motivos derivados de la evaluación forman otro conjunto (5), donde destaca que la "asistencia no es obligatoria" y no se avalúa, que vinculan la clase a la evaluación continua y esta exigen demasiado tiempo y tienen poco valor, que "optan por evaluación única" o que "existen un divorcio entre la evaluación y la docencia", o sea, entre lo que se explica y lo que de verdad de pregunta en los exámenes.

Más allá de la frecuencia de las categorías indicadas, observamos como la suma de "metodología docente" (...del profesorado) y "profesorado" recogen la frecuencia más alta con diferencia (46). Si a estas categorías hermanas le añadimos la "organización del grado" y la "evaluación" (variables que también dependen de la institución), entonces observaríamos como los factores externos al estudiante son claramente mayoritarios (60) en esta percepción cualitativa sobre los motivos del absentismo. Por su parte, las causas internas, como los motivos relacionados con las infraestructuras y la motivación y la planificación del propio estudiante, presentan una frecuencia sustancialmente menor (40).

Tabla 5: Frecuencias de motivos en análisis cualitativo: grupos y familias de causas

\begin{tabular}{|l||l|l|}
\hline Grupos de motivos & F & Familias de motivos \\
\hline \hline $\begin{array}{l}\text { Asistencia no obligatoria: no hay control o no se pasa lista y por tanto no } \\
\text { asiste a clase }\end{array}$ & 3 & Evaluación \\
\hline $\begin{array}{l}\text { Divorcio entre evaluación y docencia; evaluación inadecuada: si explica } \\
\text { algo y se pregunta otra cosa, los estudiantes que van a clase no aprueban y } \\
\text { los que no van, sí aprueban. }\end{array}$ & 1 & Evaluación \\
\hline \hline Van a evaluación única y por tanto no van a clase & 1 & Evaluación \\
\hline \hline Problemas con el transporte y distancia & 5 & Infraestucturas logística \\
\hline \hline Clases aburridas / pesadas & 5 & Metodología docente \\
\hline Clases demasiado teóricas & 1 & Metodología docente \\
\hline \hline Clases no necesarias para aprobar & 2 & Metodología docente \\
\hline Clases poco dinámicas & 2 & Metodología docente \\
\hline \hline $\begin{array}{l}\text { Disponibilidad de manuales, apuntes, pwp, etc. o de apuntes en el campus } \\
\text { virtual }\end{array}$ & 3 & Metodología docente \\
\hline
\end{tabular}




\begin{tabular}{|c|c|c|}
\hline No van por la metodología docente & 5 & Metodología docente \\
\hline El profesor lee literalmente el manual & 1 & Metodología docente \\
\hline Desmotivación de los estudiantes (interna de cada uno/a) & 4 & Motivación estudiantes \\
\hline Pereza / cansancio / sueño / climatología & 9 & Motivación estudiantes \\
\hline No me gusta el clima del aula & 1 & Motivación estudiantes \\
\hline Poco interés por parte de los estudiantes & 1 & Motivación estudiantes \\
\hline $\begin{array}{l}\text { Duración de las clases: } 2 \text { horas son demasiado pesadas, } 1,5 \text { h es poco y por } \\
1 \mathrm{~h} \text { no van }\end{array}$ & 4 & Organización del grado \\
\hline Horario: las clases empiezan demasiado pronto o demasiado tarde & 5 & Organización del grado \\
\hline $\begin{array}{l}\text { Evaluación continua de otras asignaturas: no van a clase porque tienen EC } \\
\text { de otras asignaturas }\end{array}$ & 2 & $\begin{array}{l}\text { Planificación del } \\
\text { estudiante }\end{array}$ \\
\hline Días Festivos & 2 & $\begin{array}{l}\text { Planificación del } \\
\text { estudiante }\end{array}$ \\
\hline Es más eficiente preparar la asignatura de forma autónoma que ir a clase & 2 & $\begin{array}{l}\text { Planificación del } \\
\text { estudiante }\end{array}$ \\
\hline Incompatibilidad de estudios y trabajo & 3 & $\begin{array}{l}\text { Planificación del } \\
\text { estudiante }\end{array}$ \\
\hline No van a clase para tener tiempo para estudiar & 1 & $\begin{array}{l}\text { Planificación del } \\
\text { estudiante }\end{array}$ \\
\hline Porqué cursan un doble grado & 3 & $\begin{array}{l}\text { Planificación del } \\
\text { estudiante }\end{array}$ \\
\hline Solapamientos de asignaturas y coincidencia de exámenes & 2 & $\begin{array}{l}\text { Planificación del } \\
\text { estudiante }\end{array}$ \\
\hline El profesor no se prepara las clases & 1 & Profesorado \\
\hline El profesor no explica bien & 8 & Profesorado \\
\hline El profesor no incentiva a los estudiantes & 3 & Profesorado \\
\hline El profesor no motiva a los estudiantes & 2 & Profesorado \\
\hline El profesor no planifica & 1 & Profesorado \\
\hline Poca implicación del profesor & 1 & Profesorado \\
\hline Poca motivación del profesor & 2 & Profesorado \\
\hline Calidad del profesor & 9 & Profesorado \\
\hline Problemas de salud & 2 & $\begin{array}{l}\text { Rasgos de los } \\
\text { estudiantes }\end{array}$ \\
\hline Temas personales & 3 & $\begin{array}{l}\text { Rasgos de los } \\
\text { estudiantes }\end{array}$ \\
\hline
\end{tabular}

\section{Discusión y conclusiones}

Como se ha podido observar anteriormente, existe un cierto grado de consenso a la hora de señalar los principales motivos por los que los estudiantes creen que algunos de sus compañeros no asisten a clase. Y esto es así tanto si atendemos a un análisis cuantitativo como cualitativo.

Uno de los motivos que aluden para la existencia del fenómeno del absentismo es la metodología utilizada por el profesorado, aunque se exprese de maneras diferentes. Ciertamente conviene matizar, y confirmar, esta conclusión.

Atendiendo al análisis estadístico, efectivamente "la forma de explicar del profesor" y que "las clases son pesadas" son los dos primeros motivos de manera clara y 
contundente, y que el "profesor dicta apuntes o lee transparencias" aparece en quinto lugar. Pero es que el tercer motivo es que "no se obliga a ir a clase" y el cuarto "que asisten a evaluación única”. Que no se obligue a ir a clase no es realmente un motivo, sino que hace referencia a las condiciones (no obligatoriedad) que permiten que el auténtico motivo (ej. clases aburridas) se exprese (no ir a clases). Algo parecido ocurre con la evaluación única: no es un auténtico motivo sustancial para no ir a clase, sino como una válvula o el instrumento de canaliza, vehicula o permite expresarse el auténtico motivo; la evaluación única incluso puede ser la consecuencia de no ir a clase, más que la causa. Por tanto, si descartamos estos aparentes motivos que no son motivos sustanciales, el verdadero núcleo del absentismo -según lo estiman los propios estudiantes- lo configura aquello que podemos denominar la metodología docente. El matiz del punto de vista es importante y, por supuesto, debe ser sometido a crítica.

Lo mismo se concluye también si atendemos a otras variables, igualmente bajo la óptica de análisis estadístico. Mientras apenas se perciben diferencias atendiendo al género y al turno de clases de los encuestados, en cambio se añade algún matiz según el curso y la edad, algo que está vinculado: aquí los estudiantes de último curso ya matizan los motivos: si el primer motivo sigue siendo de manera muy contundente "la forma de explicar", el segundo y el tercero ya parecen específicos de cuarto curso, como aludir a "que trabajan" y que se les "solapan los horarios", además, en cuarto lugar, de que "ya disponen de buenos apuntes". Algo muy parecido ocurre con la edad: los mayores de 24 años también consideran que "la forma de explicar" es el principal motivo sustancial para no ir a clase, pero en cambio ya no aluden enseguida a que "las clases son pesadas", sino a motivos logísticos como que "trabajan" o se les solapan los horarios de asignaturas.

Creemos que los resultados del análisis cualitativo no difieren sustancialmente de los anteriores, sino que añaden matices que en algunos casos resultan muy significativos. En cierta manera una buena lectura de las variadas respuestas abiertas de los encuestados nos sugiere que, en esencia, existen dos actores que se disputan la causalidad del absentismo: el profesorado y el estudiantado, o, lo que es lo mismo, factores externos a ellos mismos o factores internos, o todavía dicho de otra manera, la institución y "ellos". Efectivamente, todas las respuestas acerca de la evaluación, del profesor, de la metodología (del profesor) e incluso de la organización del grado, se circunscriben a "la institución" en un sentido amplio (con una frecuencia de 60 
respuestas); el "ellos" (o sea, el "yo" del estudiante) se vincula a su propia motivación, a su planificación y a las características del mismo estudiante (frecuencia de 40).

Conviene recordar que las preguntas abiertas se planteaban en el cuestionario bajo el epígrafe de "otros motivos". Pero parece evidente que no se trata de "otros" motivos, pues la mayoría ya eran recogidos en las 18 preguntas con opciones de respuesta del cuestionario, sino que el estudiante en este campo abierto ha enfatizado aquello que más le preocupaba, aquello que reiteraba como el factor principal del absentismo de sus compañeros.

Opinamos que el análisis cualitativo ha permitido enfatizar los factores causales y que dos son las ideas principales que extraemos. Por una parte que, efectivamente, la familia vinculada a la metodología docente se antoja principal. Hemos visto que las frecuencias acerca del profesorado de hecho lo eran sobre cómo actuaba el profesor, o sea, acerca de la metodología de sus clases. En el fondo, poco importa, aquí, que la metodología causante del absentismo se deba a una mala elección del profesorado o a su incapacidad (por ignorancia, desidia, poco interés, etc.) para hacerlo de otro modo. Por el motivo que sea como se impartan las clases, el caso es que la manera de impartirse motiva el abandono de las aulas. Pero además se vislumbra otro problema no menor: el divorcio entre el modelo de evaluación y la asistencia a clase. Se ha dicho que las clases no sirven para aprobar, que representan casi un estorbo para estudiar, que están desvinculadas de lo que se evalúa, que resultan difíciles de encajar con las pruebas evaluables de evaluación de continua, que no se identifican con el itinerario de evaluación única, etc.

No es ninguna novedad afirmar que la causalidad del absentismo estudiantil es compleja y que muchas variables de signo totalmente distinto, e incluso contradictorio, se entrelazan para construir una realidad nada fácil de abordar. Entre las diversas maneras de clasificar las causas, diríamos que una diferencia las causas "abordables" o susceptibles de ser abordadas con políticas y otra las causas estrictamente personales difícilmente abordables con políticas. Ante lo que hemos analizado, la Facultad o la Universidad poseen poco margen para cambios organizativos en el sentido de que implantar mayor flexibilidad horaria, por ejemplo, no reduciría de manera significativa el absentismo. Hemos visto que en general, o de manera prioritaria, no se trata de que "no puedan" asistir a clase, sino de que hay motivos vinculados a la manera de dar las clases por el profesorado que impulsan a decidir no ir. Además la institución "ofrece" 
las condiciones para no ir, puesto que la asistencia no es obligatoria y además el estudiante puede elegir el modo de evaluación única o final en lugar de la evaluación continuada que parece más vinculada a una asistencia a clase.

El núcleo del absentismo, en definitiva, se ciñe -insistimos: según los propios estudiantes- a la metodología docente. Este motivo, sin embargo, no deja de suponer una cierta abstracción, ya que se supone que los profesores desarrollan metodologías con acentos diferentes. En el fondo, no obstante, creemos que lo que se está cuestionando es aquello común que tiene la manera de desarrollar la docencia una parte importante del profesorado: la clase magistral. Ciertamente, aquella modalidad en la que el profesor explica -o, en el peor de los casos, lee apunes o transparencias- y el alumno toma apuntes.

Lo que la institución y el docente deban hacer o cómo reaccionar cuando averiguamos que las aulas se vacían por nuestra actual manera de enseñar, ya es otro debate. De lo indicado no debería inferirse automáticamente que debamos abandonar por completo la clase magistral, pero como mínimo debemos replantearnos su utilidad efectiva. Ante el eterno dilema de si de una manera u otra debemos empezar a obligar a asistir a clase o si debemos cambiar la clase para atraer al alumnado, probablemente debamos hallar soluciones híbridas e imaginativas. En todo caso estos resultados y estas reflexiones refuerzan la idea, en absoluto novedosa pero a menudo olvidada, de que debe existir una clara alineación entre objetivos de aprendizaje, metodología (esto incluye como impartir la docencia) y evaluación. Es posible que si este clásico eje estuviera bien alineado y fuera sólidamente coherente, las clases -magistrales o no- obtuvieran mayor valor añadido y en consecuencia de redujera el absentismo estudiantil.

\section{Referencias bibliográficas}

Cabrera, L., Bethencourt, J. T., González, M. \& Álvarez, P. (2006). Un estudio transversal retrospectivo sobre prolongación y abandono de estudios universitarios. Relieve, 12(1), 105-127.

Clores, M. A., (2009). A qualitive research study on school absenteeism among college students. The Asia-Pacific Education Researcher, 18, 151-165.

Devadoss, S. \& Foltz, J. (1996). Evaluation of factors influencing student class attendance and performance. American Journal of Agricultural Economics, 78(3), 499-507. 
Durden, G. C. \& Ellis, L. V. (1995). The effects of attendance on student learning in principles of economics. American Economic Review Papers and Proceedings, $85,343-346$.

Garrido, P. \& Sacristán, M. (2010). Algunos datos y reflexiones sobre las causas del absentismo estudiantil universitario [Some data and reflections on the causes of college student absenteeism. Absenteeism in university classrooms]. In J. L. Jiménez \& A. Rodríguez (Eds.), El absentismo en las aulas universitarias. El caso de la Escuela Universitaria de Estudios Empresariales de la Universidad de Sevilla [The case of the School of Business Studies at the University f Seville] (pp. 151-168). Granada: Grupo Editorial Universitario.194J.M. López-Bonilla and L.M. López-Bonilla Downloaded by [Society for Research into Higher Education SRHE] at 01:38 27 September 2017.

GESOP (2008). Absentisme i abandonament entre els estudiants de primer curs de la Facultat de Dret (UB). Informe de Resultats.

GESOP (2010). Absentisme entre els alumnes de nou ingrés al grau de Dret de la UB. Informe de resultats.

Gracia, E., \& Iglesia, M. C. (2007). Absentismo entre los estudiantes de Teoría Económica. Un análisis cuantitativo [Absenteeism among students of economic theory. Quantitative analysis]. In D. Dávila-Quintana, S. Rodríguez-Feijoó, M. Tejera-Gil, Y. Santana-Jiménez, J. A. Gil-Jurado \& A. Rodríguez-Caro (Eds.), Investigaciones de Economía de la Educación [Research on economics of education] (pp. 231-240). Madrid: AEDE y Delta Publicaciones.

Halpern, N. (2007). Attendance in Higher Education: does it matter? Investigations in university teaching and learning, 4(2), 7-13.

Longhurst, R. J. (1999). Why aren't they here? Student absenteeism in a further education college. Journal of Further \& Higher Education, 23, 61-80.

López-Bonilla, J. M. \& López-Bonilla, L. M. (2015). The multidimensional structure of university absenteeism: an exploratory study. Innovations in Education and Teaching International, 52(2), 185-195.

McCarey, M., Barr, T. \& Rattray, J. (2007). Predictors of academic performance in a cohort of pre-registration nursing students. Nurse Education Today, 27(4), 357364.

Marburger, D. R. (2001). Absenteeism and undergraduate exam performance. The Journal of Economic Education, 32(2), 99-109.

Marburger, D. R. (2006). Does mandatory attendance improve student performance? The Journal of Economic Education, 37, 148-155.

Moore, S., Armstrong, C. and Pearson, J. (2008) Lecture absenteeism among students in higher education: a valuable route to understanding student motivation, Journal of Higher Education Policy and Management, 30 (1), 15-24. 
Paisey, C. \& Paisey, N. J. (2004). Student attendance in an accounting module-reasons for non-attendance and the effect on academic performance at a Scottish University. Accounting education, 13 (sup1), 39-53.

Reid, K. (2008). The causes of non-attendance: An empirical study. Educational Review, 60,345-357.

Rodríguez González, R., Hernández García, J., Alonso Gutiérrez, A. M. \& Díez Itza, E. (2003). El absentismo en la Universidad: resultados de una encuesta sobre motivos que señalan los estudiantes para no asistir a clase. Aula Abierta, 82.

Rodríguez, A. (2010). Causas del absentismo según los estudiantes [Causes of absenteeismby students]. In J. L. Jiménez \& A. Rodríguez, (Eds.), El absentismo en las aulas univer-sitarias. El caso de la Escuela Universitaria de Estudios Empresariales de la Universi-dad de Sevilla [The case of the School of Business Studies at the University of Seville] (pp. 101-124). Granada: Grupo Editorial Universitario.

Romer, D. (1993). Do students go to class? Should they? The Journal of Economic Perspectives, 7(3), 167-174.

Ruiz, A., Ceballos, C., \& García, J. A. (2010). Utilización de un sistema de respuesta interactiva como herramienta contra el absentismo en la Universidad. En: J. L. Jiménez \& A. Rodríguez (Eds.), El absentismo en las aulas universitarias. El caso de la Escuela Universitaria de Estudios Empresariales de la Universidad de Sevilla. (pp. 179-192). Granada: Grupo Editorial Universitario.

Triadó-Ivern, X., Aparicio-Chueca, P., Guàrdia-Olmos, J. \& Jaría-Chacón, N. (2009a). Aproximación empírica al análisis del absentismo de los estudiantes universitarios. Estudio del caso de la Facultad de Economía y Empresa (UB) Revista de Formación e Innovación Educativa Universitaria, 2, 182-192.

Triadó-Ivern, X., Aparicio-Chueca, P., Guàrdia-Olmos, J., Peró-Cebollero, M. \& JaríaChacón, N. (2013). Empirical approach to the analysis of university student absenteeism: proposal of a questionnaire for students to evaluate the possible causes. Quality \& Quantity, 1-8.

Turull, M; Roca, B. (2012). Per què no van a classe els estudiants? Un estudi de camp sobre les causes de l'absentisme acadèmic dels estudiants de primer curs de Dret de la UB. Universidad de Barcelona, Facultad de Derecho (recurso on line: http://hdl.handle.net/2445/27250 ). 